\title{
FRACTAL PROPERTIES OF RANDOM VARIABLES WITH INDEPENDENT $Q_{\infty}$-SYMBOLS
}

UDC 519.21

\author{
R. O. NIKIFOROV AND G. M. TORBIN
}

\begin{abstract}
We study an equivalent definition of the Hausdorff-Besicovitch dimension in terms of a system $\Phi\left(Q_{\infty}\right)$ of cylinders of the $Q_{\infty}$-expansion. Sufficient conditions for the system $\Phi\left(Q_{\infty}\right)$ to be faithful for the evaluation of the Hausdorff-Besicovitch dimension in the unit interval are found; fine fractal properties of probability measures with independent $Q_{\infty}$-digits are investigated (we do not assume that the $Q_{\infty^{-}}$ digits are identically distributed).
\end{abstract}

\section{INTRODUCTION}

This paper is devoted to a study of fractal properties of one-dimensional singular probability measures with independent $Q_{\infty}$-symbols. We briefly recall below the notion of the $Q_{\infty}$-expansion of real numbers and some other definitions of the $Q_{\infty}$-expansion (see 24] for more details). Let $Q_{\infty}=\left(q_{0}, q_{1}, q_{2}, \ldots\right)$ be an infinite stochastic vector with positive coordinates. Given a vector $Q_{\infty}$ we construct an infinite sequence of partitions of the unit interval according to the following rules.

Step 1. We split the set $[0,1)$ into an infinite number of disjoint intervals $\Delta_{i_{1}}^{Q_{\infty}}, i_{1} \in$ $\{0,1,2, \ldots\}$, of the form $[a, b)$,

$$
[0,1)=\bigcup_{i_{1}=0}^{\infty} \Delta_{i_{1}}^{Q_{\infty}} .
$$

The length of an interval $\Delta_{i_{1}}^{Q_{\infty}}$ is equal to $q_{i_{1}}$ and denoted by $\left|\Delta_{i_{1}}^{Q_{\infty}}\right|$.

Each interval $\Delta_{i_{1}}^{Q \infty}$ is called a cylinder of the first rank for the $Q_{\infty}$-expansions.

Step $k \geq 2$. Each cylinder $\Delta_{i_{1} i_{2} \ldots i_{k-1}}^{Q_{\infty}}$ of rank $(k-1)$ is partitioned into an infinite number of disjoint intervals $\Delta_{i_{1} i_{2} \ldots i_{k}}^{Q_{\infty}}$ of the form $[a, b)$,

$$
\Delta_{i_{1} i_{2} \ldots i_{k-1}}^{Q_{\infty}}=\bigcup_{i_{k}=0}^{\infty} \Delta_{i_{1} i_{2} \ldots i_{k}}^{Q_{\infty}} .
$$

The lengths of the intervals

$$
\left|\Delta_{i_{1} i_{2} \ldots i_{k}}^{Q_{\infty}}\right|=q_{i_{1}} \cdot q_{i_{2}} \cdots q_{i_{k}}=\prod_{s=1}^{k} q_{i_{s}}
$$

2010 Mathematics Subject Classification. Primary 60G30, 11K55, 28A80.

Key words and phrases. $Q_{\infty}$-expansions, faithful systems of coverings, singularly continuous probability distributions, Hausdorff-Besicovitch dimension of a set, Hausdorff dimension of a measure.

The first author was supported by the Project DFG 436113/97.

The second author was supported by the Projects DFG 436 UKR 113/97 and DFG KO 1989/6-1 and the Alexander von Humboldt Foundation. 
are such that

$$
\left|\Delta_{i_{1} i_{2} \ldots i_{k-1} 0}^{Q_{\infty}}\right|:\left|\Delta_{i_{1} i_{2} \ldots i_{k-1} 1}^{Q_{\infty}}\right|: \ldots:\left|\Delta_{i_{1} i_{2} \ldots i_{k-1} m}^{Q_{\infty}}\right|: \ldots=q_{0}: q_{1}: \ldots: q_{m}: \ldots
$$

Each interval $\Delta_{i_{1} i_{2} \ldots i_{k}}^{Q_{\infty}}$ is called a cylinder of rank $k$ for the $Q_{\infty}$-expansion.

For an arbitrary sequence of indices $\left\{i_{k}\right\}, i_{k} \in\{0,1,2 \ldots\}$, there exists a sequence of decreasing cylinders

$$
\Delta_{i_{1}}^{Q_{\infty}} \supset \Delta_{i_{1} i_{2}}^{Q_{\infty}} \supset \cdots \supset \Delta_{i_{1} i_{2} \cdots i_{k}}^{Q_{\infty}} \supset \cdots
$$

such that $\left|\Delta_{i_{1} \ldots i_{k}}^{Q_{\infty}}\right| \rightarrow 0$ as $k \rightarrow \infty$. This means that there exists a unique point $x \in[0,1)$ that belongs to all of the cylinders $\Delta_{i_{1}}^{Q_{\infty}}, \Delta_{i_{1} i_{2}}^{Q_{\infty}}, \ldots, \Delta_{i_{1} i_{2} \ldots i_{k}}^{Q_{\infty}}, \ldots$

Conversely, for every point $x \in[0,1)$, there exists a unique sequence of decreasing cylinders $\Delta_{i_{1}(x)}^{Q_{\infty}} \supset \Delta_{i_{1}(x) i_{2}(x)}^{Q_{\infty}} \supset \cdots \supset \Delta_{i_{1}(x) i_{2}(x) \ldots i_{k}(x)}^{Q_{\infty}} \supset \ldots$ such that

$$
x=\bigcap_{k=1}^{\infty} \Delta_{i_{1}(x) i_{2}(x) \ldots i_{k}(x)}^{Q_{\infty}}=: \Delta_{i_{1}(x) i_{2}(x) \ldots i_{k}(x) \ldots}^{Q_{\infty} \ldots} .
$$

The uniqueness of such a sequence of cylinders is explained by the property that, given a point of the interval $[0,1)$, there exists a unique cylinder of rank $n$ that contains this point.

The symbol on the right-hand side of the latter formula is called the $Q_{\infty}$-expansion of a point $x$. The basic results of the metric and probabilistic theory of $Q_{\infty}$-expansions were developed by M. V. Pratsevytyı in 1990 and are presented in the monographs [18. and [24]. The notion of a $Q_{\infty}$-expansion (as well as a $Q_{\infty}$-representation) was introduced by Pratsevytyı [18, 24].

Note that a $Q_{\infty}$-expansion of real numbers is a particular case of the so-called $f$ expansion of real numbers (see [12, 22]) with continuous increasing functions $f$ defined in the semi-axis $[0,+\infty)$. A $Q_{\infty}$-expansion corresponds to the function $f$ that linearly increases in the intervals $[n, n+1]$ such that $f(0)=0$ and $f(n+1)-f(n)=q_{n}$ for all $n \in\{0,1,2, \ldots\}$.

Note also that a $Q_{\infty}$-expansion can be defined via a system of iterating functions (see [13, 14]). Indeed, consider the system of iterating functions generated by the following denumerable system of contracting transformations:

$$
F_{0}(x)=q_{0} \cdot x, \quad F_{i}(x)=q_{i} \cdot x+\left(q_{0}+\cdots+q_{i-1}\right), \quad \forall i \in \mathbf{N} .
$$

It is clear that the set $[0,1)$ is invariant with respect to this system of iterating functions, since $[0,1)=\bigcup_{i=0}^{\infty} F_{i}([0,1))$. Moreover, the cylinders of the $Q_{\infty}$-expansion defined above are such that

$$
\Delta_{i_{1} i_{2} \ldots i_{k}}^{Q_{\infty}}=F_{i_{1}} \circ F_{i_{2}} \circ \cdots \circ F_{i_{k}}([0,1)) .
$$

Thus, given an arbitrary $x \in[0,1)$, there exists a unique sequence of integers $\left(i_{1}(x), i_{2}(x), \ldots, i_{k}(x), \ldots\right) \in\{0,1,2, \ldots\}^{\infty}$ such that

$$
x=\bigcap_{k=1}^{\infty} F_{i_{1}} \circ F_{i_{2}} \circ \cdots \circ F_{i_{k}}([0,1))=\Delta_{i_{1}(x) i_{2}(x) \ldots i_{k}(x) \ldots}^{Q_{\infty}} .
$$

The normal properties of real numbers (that is, those properties that hold for almost all (with respect to the Lebesgue measure) real numbers of the interval $[0,1)$ ) expressed in terms of their $Q_{\infty}$-expansions are studied in the papers [16, 24, 18]. In particular, it is known that the asymptotic density of a symbol $i$ of the alphabet $A=\{0,1,2, \ldots\}$ in $Q_{\infty}$-expansions of almost all (with respect to the Lebesgue measure) real numbers belonging to the unit interval is equal to $q_{i}$ (see [18]). If the entropy of a stochastic 
vector $Q_{\infty}$ is finite for almost all (with respect to the Lebesgue measure) real numbers belonging to the unit interval, then

$$
\lim _{n \rightarrow \infty} \sqrt[n]{\left|\Delta_{i_{1}(x) \ldots i_{n}(x)}^{Q_{\infty}}\right|}=e^{-H}
$$

where $H=-\sum_{i=0}^{\infty} q_{i} \ln q_{i}($ see [16]).

Some other phenomena related to $Q_{\infty}$-expansions and fractal analysis are discussed in 2 .

Recall that a family $\Phi$ of subsets of the interval $[0,1)$ is called a locally fine family of coverings if, for an arbitrary set $E \subset[0,1)$ and an arbitrary number $\varepsilon>0$, there exists an at most denumerable $\varepsilon$-covering $\left\{E_{j}\right\}$ of the set $E$, where $E_{j} \in \Phi$ are such that $\left|E_{j}\right| \leq \varepsilon$ (see [21]). Given an arbitrary positive $\alpha$ and a locally fine family of coverings $\Phi$, the corresponding $\alpha$-dimensional Hausdorff measure of a subset $E \subset[0,1)$ related to the family $\Phi$ is defined by

$$
H^{\alpha}(E, \Phi)=\lim _{\varepsilon \rightarrow 0}\left[\inf _{\left|E_{j}\right| \leq \varepsilon}\left\{\sum_{j}\left|E_{j}\right|^{\alpha}\right\}\right],
$$

where inf is considered with respect to all at most denumerable $\varepsilon$-coverings $\left\{E_{j}\right\}$ of the set $E, E_{j} \in \Phi$.

In general, $H^{\alpha}(E, \Phi)$ depends on a family $\Phi$. However, the family of all bounded sets, the family of all open sets, and the family of all closed sets generate the same $\alpha$-dimensional Hausdorff measure (see [13). This measure is denoted by $H^{\alpha}(E)$.

Definition 1.1. A nonnegative number

$$
\operatorname{dim}_{H}(E, \Phi)=\inf \left\{\alpha: H^{\alpha}(E, \Phi)=0\right\}
$$

is called the Hausdorff-Besicovitch dimension of the set $E$ with respect to the family of subsets $\Phi$.

If $\Phi$ is the family of all closed (open) subsets of $[0,1]$ or the family of all $s$-adic cylinders, then $\operatorname{dim}_{H}(E, \Phi)$ coincides with the classical Hausdorff-Besicovitch dimension $\operatorname{dim}_{H}(E)$ of a set $E$ (see [7]).

Definition 1.2. A locally fine family of coverings $\Phi$ is called faithful for the evaluation of the Hausdorff-Besicovitch dimension in the unit interval if

$$
\operatorname{dim}_{H}(E, \Phi)=\operatorname{dim}_{H}(E)
$$

for an arbitrary set $E \subset[0,1]$.

The family of all subsets of the unit interval, the family of all subintervals, and the family of $s$-adic cylinders are examples of faithful families for the evaluation of the HausdorffBesicovitch dimension in the unit interval. Sufficient conditions for families of subsets to be faithful are studied by many authors (see, for example, 11, 2, 7, 11, 18, 21, 24. and references therein). It is surprising that the first examples of families that are not faithful appeared in 1990 for the two-dimensional case as a result of the study of fractal properties of self-affine sets (see [6]). As far as the authors of this paper are aware, the first example of a one-dimensional locally fine family of coverings that is not faithful is constructed in 17] (this family coincides with the family of cylinders of the classical chain representation). The approach introduced by Yuval Peres is used in [2] to prove that a family of cylinders of the chain representation is not faithful. This result is established in [2] by using the fact that the family of cylinders of $Q_{\infty}$-expansions is not faithful if 
the order of decrease of the sequence $\left\{q_{i}\right\}$ is polynomial; in other words, if

$$
\frac{A}{(i+1)^{s}} \leq q_{i} \leq \frac{B}{(i+1)^{s}}
$$

for some positive constants $A$ and $B$, where $s>1$. Sufficient conditions are found in 2 for such families to be not faithful.

The main aim of the current paper is to find sufficient conditions for the family of cylinders of $Q_{\infty}$-expansions to be faithful (Section 2) and then to apply these conditions to the study of fine fractal properties of probability measures with independent $Q_{\infty}$-digits (Section 3). In other words, we study the distributions of the random variables

$$
\xi=\Delta_{\xi_{1} \xi_{2} \ldots \xi_{k} \ldots}^{Q}
$$

where $\xi_{k}$ are independent random variables assuming values in the set $A=\{0,1,2, \ldots\}$ with probabilities $p_{0 k}, p_{1 k}, p_{2 k}, \ldots$, respectively.

\section{2. $Q_{\infty}$-REPRESENTATIONS OF REAL NUMBERS AND FAITHFUL COVERING FAMILIES IN THE UNIT INTERVAL}

The evaluation of the Hausdorff-Besicovitch is a nontrivial problem even for onedimensional sets. A way to make it more feasible is to reduce the class of admissible coverings to a certain locally fine family of coverings $\Phi$ being faithful. This idea is especially helpful if one follows Billingsley's approach [7, 8]. Therefore, an important problem of the fractal analysis is to check whether or not a family of coverings is faithful, or to find sufficient conditions for a family of coverings to be faithful.

The first results of this kind belong to Besicovitch [5] who proved that the family of cylinders for the dyadic expansion is faithful. Billingsley [7] extended this result to the family of $s$-adic cylinders. Pratsevyty $\breve{1} 24$ obtained the same result for the family of cylinders of $Q-S$-expansions of real numbers. The method of investigation used in these papers is the same and consists in finding a positive constant $K \in N$ such that, for an arbitrary positive $\varepsilon$ and of an arbitrary interval $[a, b]$, there exist at most $K$ cylinders of the corresponding family that covers $[a, b]$, and that the diameter of each of the cylinders does not exceed $\varepsilon$.

It is clear that this method does not apply to the family of cylinders of $Q_{\infty}$-expansions. Some new results concerning the family of cylinders of $Q_{\infty}$-expansions are presented in this section. The results below generalize those in [3, 16. The method of proof is also different from those in [3, 16.

In what follows we restrict consideration to the case of cylinders of $Q_{\infty}$-expansions. So that no confusion may occur, we suppress the upper index $Q_{\infty}$. As the family $\Phi$, we understand the family $\Phi\left(Q_{\infty}\right)$ of cylinders of all possible ranks of a $Q_{\infty}$-partition of the interval $[0,1)$, that is,

$$
\Phi=\left\{E: E=\Delta_{\alpha_{1} \ldots \alpha_{n}}, n \in \mathbf{N}, \alpha_{i} \in \mathbf{N} \cup 0, i=1,2, \ldots, n\right\} .
$$

Theorem 2.1. If there exist two numbers $c_{1}$ and $c_{2}$ such that

$$
0<c_{1} \leq \frac{q_{i}}{q_{i-1}} \leq c_{2}<1, \quad \forall i \in \mathbf{N}
$$

then the system $\Phi$ of cylinders of a $Q_{\infty}$-expansion is faithful for the evaluation of the Hausdorff-Besicovitch dimension. 
Proof. Let

$$
\begin{aligned}
& H_{\varepsilon}^{\alpha}(E)=\inf _{\left|E_{j}\right| \leq \varepsilon}\left\{\sum_{j}\left|E_{j}\right|^{\alpha}, E_{j}=\left[a_{j}, b_{j}\right], E \subset \bigcup_{j} E_{j}\right\}, \\
& H_{\varepsilon}^{\alpha}(E, \Phi)=\inf _{\left|B_{j}\right| \leq \varepsilon}\left\{\sum_{j}\left|B_{j}\right|^{\alpha}, B_{j} \in \Phi, E \subset \bigcup_{j} B_{j}\right\} .
\end{aligned}
$$

It is obvious that $H_{\varepsilon}^{\alpha}(E) \leq H_{\varepsilon}^{\alpha}(E, \Phi)$, since the first infimum is evaluated with respect to a wider class of admissible coverings.

Let $E_{j}=\left[a_{j}, b_{j}\right]$. By $\Delta_{\left[m_{j}-1\right]}$ we denote a $Q_{\infty}$-cylinder of a maximal rank that contains both points $a_{j}$ and $b_{j}$ (it is possible that the cylinder $\Delta_{\left[m_{j}-1\right]}$ coincides with the whole interval $[0,1))$. By $\Delta_{\left[m_{j}\right]}^{i}$ we denote the cylinders of rank $m_{j}$ that belong to the cylinder $\Delta_{\left[m_{j}-1\right]}$, that is,

$$
\Delta_{\left[m_{j}-1\right]}=\bigcup_{i=0}^{\infty} \Delta_{\left[m_{j}\right]}^{i} .
$$

The points $a_{j}$ and $b_{j}$ belong to different cylinders of rank $m_{j}$. We distinguish between two possible cases.

I) If there exists at least one cylinder of rank $m_{j}$ that belongs to the interval $\left[a_{j}, b_{j}\right]$, then there is a cylinder $\Delta_{\left[m_{j}\right]}^{k_{j}}$ that belongs to $\left[a_{j}, b_{j}\right]$ and either $a_{j}=\inf \Delta_{\left[m_{j}\right]}^{k_{j}}$ or $a_{j} \in$ $\Delta_{\left[m_{j}\right]}^{k_{j}-1}$. Then $E_{j} \subset \bigcup_{i=k_{j}-1}^{\infty} \Delta_{\left[m_{j}\right]}^{i}$ and $\left|\Delta_{\left[m_{j}\right]}^{i}\right| \leq \frac{1}{c_{1}} \varepsilon$. Moreover,

$$
\sum_{i=k_{j}-1}^{\infty}\left|\Delta_{\left[m_{j}\right]}^{i}\right|^{\alpha} \leq\left|\Delta_{\left[m_{j}\right]}^{k_{j}}\right|^{\alpha}\left(\frac{1}{c_{1}^{\alpha}}+\sum_{i=0}^{\infty} c_{2}^{\alpha i}\right) \leq\left|E_{j}\right|^{\alpha}\left(\frac{1}{c_{1}^{\alpha}}+\frac{1}{1-c_{2}^{\alpha}}\right) .
$$

II) If there are no cylinders of rank $m_{j}$ that belong to the interval $\left[a_{j}, b_{j}\right]$, then the points $a_{j}$ and $b_{j}$ belong to two adjacent cylinders, $\Delta_{\left[m_{j}\right]}^{s_{j}}$ and $\Delta_{\left[m_{j}\right]}^{s_{j}+1}$.

Let $\Delta_{\left[m_{j}+1\right]}^{l_{j}}$ be the cylinder of rank $m_{j}+1$ that contains the point $a_{j}$ and let $M_{j}=$ $\sup \Delta_{\left[m_{j}\right]}^{s_{j}}$. Then

$$
\begin{gathered}
\bigcup_{i=l_{j}}^{\infty} \Delta_{\left[m_{j}+1\right]}^{i} \supset\left[a_{j}, M_{j}\right), \quad \Delta_{\left[m_{j}+1\right]}^{l_{j}+1} \subset\left[a_{j}, M_{j}\right), \\
\left|\Delta_{\left[m_{j}+1\right]}^{i}\right| \leq \frac{1}{c_{1}}\left|E_{j}\right|, \quad \forall i \geq l_{j} .
\end{gathered}
$$

Thus,

$$
\begin{aligned}
\sum_{i=l_{j}}^{\infty}\left|\Delta_{\left[m_{j}+1\right]}^{i}\right|^{\alpha} & \leq\left|\Delta_{\left[m_{j}+1\right]}^{l_{j}}\right|^{\alpha}+\left|\Delta_{\left[m_{j}+1\right]}^{l_{j}+1}\right|^{\alpha}+c_{2}^{\alpha}\left|\Delta_{\left[m_{j}+1\right]}^{l_{j}+1}\right|^{\alpha}+c_{2}^{2 \alpha}\left|\Delta_{\left[m_{j}+1\right]}^{l_{j}+1}\right|^{\alpha}+\ldots \\
& \leq\left|\Delta_{\left[m_{j}+1\right]}^{l_{j}+1}\right|^{\alpha}\left(\frac{1}{c_{1}^{\alpha}}+\sum_{k=0}^{\infty} c_{2}^{\alpha k}\right) \leq\left|E_{j}\right|^{\alpha}\left(\frac{1}{c_{1}^{\alpha}}+\frac{1}{1-c_{2}^{\alpha}}\right) .
\end{aligned}
$$

Since $M_{j}$ is a point of the $Q_{\infty}$-covering, $M_{j}$ is the left end point for a sequence of nested cylinders. The sequence of these nested cylinders is denoted by

$$
\Delta_{\left[m_{j}+1\right]}, \quad \Delta_{\left[m_{j}+2\right]}, \ldots .
$$

Let $\Delta_{\left[m_{j}+t_{j}\right]}$ be a cylinder of rank $m_{j}+t_{j}$ such that $b_{j} \notin \Delta_{\left[m_{j}+t_{j}\right]}$ and

$$
b_{j} \in \Delta_{\left[m_{j}+t_{j}-1\right]} \text {. }
$$


Then

$$
\begin{gathered}
\left|\Delta_{\left[m_{j}+t_{j}\right]}\right| \leq\left|b_{j}-M_{j}\right| \leq\left|E_{j}\right|, \\
\left|\Delta_{\left[m_{j}+t_{j}-1\right]}\right|=\frac{1}{q_{0}}\left|\Delta_{\left[m_{j}+t_{j}\right]}\right| \leq \frac{1}{q_{0}}\left|E_{j}\right| .
\end{gathered}
$$

Note that $\Delta_{\left[m_{j}+t_{j}-1\right]}$ covers $\left[M_{j}, b_{j}\right]$.

Hence the family of cylinders $\Delta_{\left[m_{j}+1\right]}^{l_{j}}, \Delta_{\left[m_{j}+1\right]}^{l_{j}+1}, \ldots$ and $\Delta_{\left[m_{j}+t_{j}-1\right]}$ covers $E_{j}$ and the lengths of these cylinders do not exceed $\left|E_{j}\right| \cdot \max \left\{1 / c_{1}, 1 / q_{0}\right\}=\left|E_{j}\right| \cdot k_{0}$. Moreover, their $\alpha$-volume does not exceed

$$
\left|E_{j}\right|^{\alpha}\left(\frac{1}{c_{1}^{\alpha}}+\frac{1}{1-c_{2}^{\alpha}}+\frac{1}{q_{0}^{\alpha}}\right) .
$$

This means that an arbitrary interval $E_{j}$ can be covered by cylinders of the family $\Phi$ such that the diameters of the cylinders do not exceed $k_{0} \varepsilon$, where $k_{0}:=\max \left\{1 / c_{1}, 1 / q_{0}\right\}$. Moreover, given an arbitrary $\alpha \in(0,1]$, the corresponding $\alpha$-volume of this covering does not exceed $F(\alpha)\left|E_{j}\right|^{\alpha}$, where

$$
F(\alpha)=\left(\frac{1}{c_{1}^{\alpha}}+\frac{1}{1-c_{2}^{\alpha}}+\frac{1}{q_{0}^{\alpha}}\right) .
$$

Therefore,

$$
H_{k_{0} \varepsilon}^{\alpha}(E, \Phi) \leq F(\alpha) \sum_{j}\left|E_{j}\right|^{\alpha}
$$

for an arbitrary $\varepsilon>0$, for all $\alpha \in(0,1]$, for all sets $E \subset[0,1)$, and an arbitrary $\varepsilon$-covering $\left\{E_{j}\right\}$ of the set $E$. Thus, given an arbitrary $\varepsilon>0$, we have

$$
H_{k_{0} \varepsilon}^{\alpha}(E, \Phi) \leq F(\alpha) H_{\varepsilon}^{\alpha}(E)
$$

for all $\alpha \in(0,1]$ and all sets $E \subset[0,1)$. Passing to the limit as $\varepsilon \rightarrow 0$, we get

$$
H^{\alpha}(E) \leq H^{\alpha}(E, \Phi) \leq F(\alpha) H^{\alpha}(E)
$$

for all $\alpha \in(0,1]$ and an arbitrary $E \subset[0,1)$. This proves that

$$
\operatorname{dim}_{H}(E, \Phi)=\operatorname{dim}_{H}(E), \quad \forall E \subset[0,1) .
$$

\section{Fine fractal properties of probability measures \\ WITH INDEPENDENT $Q_{\infty}$-DIGITS}

Let $\left\{\xi_{k}\right\}$ be a sequence of independent random variables assuming values $0,1,2, \ldots$ with probabilities $p_{0 k}, p_{1 k}, p_{2 k}, \ldots$, respectively, where $\left\{p_{i k}\right\}$ are such that

$$
\sum_{i=0}^{\infty} p_{i k}=1 \quad \text { for all } k \in \mathbf{N} .
$$

Consider the random variable

$$
\xi:=\Delta_{\xi_{1}} \xi_{2} \ldots \xi_{k} \ldots,
$$

called a random variable with independent $Q_{\infty}$-digits. The probability distribution $\mu_{\xi}$ determined by a stochastic vector $Q_{\infty}$ and a matrix $P=\left\|p_{i k}\right\|$ can be constructed as follows. Let $\Omega_{k}=\{0,1,2, \ldots\}, \mathcal{F}_{k}=2^{\Omega_{k}}, \mu_{k}(i)=p_{i k},(\Omega, \mathcal{F}, \mu)=\prod_{i=1}^{\infty}\left(\Omega_{k}, \mathcal{F}_{k}, \mu_{k}\right)$, and let $f: \Omega \rightarrow[0,1)$ be a measurable mapping defined by $f(\omega)=\Delta_{\omega_{1} \omega_{2} \ldots \omega_{i} \ldots}$ for an arbitrary $\omega=\left(\omega_{1}, \omega_{2}, \ldots, \omega_{i}, \ldots\right) \in \Omega$. For an arbitrary Borel set $E \subset[0,1)$, we introduce the image measure $\mu^{*}$ by

$$
\mu^{*}(E)=\mu\left(f^{-1}(E)\right),
$$

where $f^{-1}(E)=\{\omega: \omega \in \Omega, f(\omega) \in E\}$. Then the image measure $\mu^{*}$ coincides with the probability measure $\mu_{\xi}$. 
If the discrete measures $\lambda_{k}$ are such that $\lambda_{k}(i)=q_{i}$ for all $k \in N \cup\{0\}$, and

$$
(\Omega, \mathcal{F}, \lambda)=\prod_{k=1}^{\infty}\left(\Omega_{k}, \mathcal{F}_{k}, \lambda_{k}\right)
$$

then the image measure $\lambda^{*}=\lambda\left(f^{-1}\right)$ coincides with the Lebesgue measure in the interval $[0,1)$.

It is known that the distribution of the random variable $\xi$ is of a pure Lebesgue type (see [16]). Moreover,

1) $\mu_{\xi}$ is a pure absolutely continuous measure if and only if

$$
\rho:=\prod_{k=1}^{\infty}\left\{\sum_{i=0}^{\infty} \sqrt{p_{i k} \cdot q_{i}}\right\}>0
$$

2) $\mu_{\xi}$ is a pure discrete measure if and only if

$$
P_{\max }:=\prod_{k=1}^{\infty} \max _{i}\left\{p_{i k}\right\}>0
$$

3) otherwise $\mu_{\xi}$ is a pure singularly continuous measure; this is the case if

$$
\rho=0=P_{\max } \text {. }
$$

Other criteria for the absolute continuity or singularity of the measure $\mu_{\xi}$ can be found in 18 .

It is easy to see that a necessary condition that $\mu_{\xi}$ is absolutely continuous is the "asymptotic compatibility" of the matrices $P_{\infty}$ and $Q_{\infty}$. In other words, a necessary condition is that $p_{i k} \rightarrow q_{i}$ as $k \rightarrow \infty$ for all $i \in N_{0}$. A necessary condition that $\mu_{\xi}$ is discrete is that $\max _{i} p_{i k} \rightarrow 1$ as $k \rightarrow \infty$. In all the remaining cases the distribution of $\mu_{\xi}$ is singularly continuous. This indicates that, in the sense explained above, the property of singularity dominates in this class of probability measures. According to the multiscale fractal analysis of singular probability measures proposed in [23], after the singularity is proved one proceeds to the investigation of topological-metric and fractal properties of the spectrum $S_{\mu}$ of the distribution and of fine fractal properties of the measure. Recall that the spectrum of a distribution is its minimal closed support.

Recall (see [3]) that a singularly continuous probability measure $\mu$ in $\mathbf{R}^{1}$ is said to belong to the pure

a) $G S$-type if there is a sequence of (nonoverlapping) intervals $\left\{\left[a_{i}, b_{i}\right]\right\}$ such that

$$
\left\{\begin{array}{l}
{\left[a_{i}, b_{i}\right] \subset S_{\mu}} \\
\mu\left(\bigcup_{i}\left[a_{i}, b_{i}\right]\right)=1
\end{array}\right.
$$

b) $G C$-type if there exists a nowhere dense set $E$ such that

$$
\left\{\begin{array}{l}
E \subset S_{\mu} \\
\mu(E)=1 \\
\forall x \in E \exists \varepsilon(x)>0:[x-\varepsilon(x), x+\varepsilon(x)] \cap S_{\mu} \text { is a Lebesgue null set. }
\end{array}\right.
$$

c) $G P$-type if there exists a nowhere dense set $E$ such that

$$
\left\{\begin{array}{l}
E \subset S_{\mu} \\
\mu(E)=1 \\
\forall x \in E \forall \varepsilon>0:[x-\varepsilon, x+\varepsilon] \cap S_{\mu} \text { is a Lebesgue null set. }
\end{array}\right.
$$

Singularly continuous measures of GC-, GP-, and GS-types constitute disjoint families. The union of these families does not coincide with the family of all singularly continuous probability measures in $R^{1}$, since there exist singularly continuous probability measures in $R^{1}$ that do not belong to any of the above classes. On the other hand, it is proved 
in 20] that every singularly continuous probability measure $\mu$ in $R^{1}$ is represented in the form

$$
\mu=\alpha_{1} \mu^{G S}+\alpha_{2} \mu^{G C}+\alpha_{3} \mu^{G P},
$$

where $\alpha_{1} \geq 0, \alpha_{2} \geq 0, \alpha_{3} \geq 0, \alpha_{1}+\alpha_{2}+\alpha_{3}=1$, and where $\mu^{G S}, \mu^{G C}$, and $\mu^{G P}$ are singularly continuous probability measures of $G S$-, $G C$-, and $G P$-type, respectively.

It is known (see [16]) that every singularly continuous random variable with independent $Q_{\infty}$-symbols is of a pure topological-metric type. Moreover,

1) $\mu_{\xi}$ is of a pure GS-type if and only if the matrix $P$ contains at most finite number columns with zero entries;

2) $\mu_{\xi}$ is of a pure GC-type if and only if the matrix $P$ contains an infinite number of columns with zero elements and

$$
\sum_{k=1}^{\infty}\left(\sum_{i: p_{i k}=0} q_{i}\right)=\infty ;
$$

3) $\mu_{\xi}$ is of a pure GP-type if and only if the matrix $P$ contains an infinite number of columns with zero elements and

$$
\sum_{k=1}^{\infty}\left(\sum_{i: p_{i k}=0} q_{i}\right)<\infty .
$$

Remark 3.1. Another approach (which is not equivalent to the one discussed above) to classify singular measures is proposed in the monograph [18. An essential ingredient of both approaches is the study of topological and metric properties of the spectrum of distributions of random variables with independent $Q_{\infty}$-symbols. The basic results of this type are obtained in 18 .

It is worth mentioning that similar problems concerning the Lebesgue structure and topological metric properties of the spectrum are considered in [19] for random variables with independent $\widetilde{Q}_{\infty}$-symbols. The $\widetilde{Q}_{\infty}$-expansion of a real number is similar to the $Q_{\infty}$-expansion with the exception that the cylinders of odd ranks are partitioned by the cylinders of the next rank in the direction from left to right, while the cylinders of even ranks are partitioned by the cylinders of the next rank in the direction from right to left (see [19, 24] for details).

The $\widetilde{Q}_{\infty}$-expansion of real numbers is a particular case of the so-called $f$-expansions introduced by Bissinger [9] and is a generalization of continued fractions. The $\widetilde{Q}_{\infty^{-}}$ expansion corresponds to the decreasing continuous function $f$ defined in the semiaxis $[1,+\infty)$ as follows: $f(1)=1, f(n)-f(n+1)=q_{n-1}$ for all $n \in \mathbf{N}$, and $f$ is linear in every interval $[n, n+1]$. Bissinger [9] uses the notation $F_{p}$ for such a class of functions. Some metric theory of such decompositions is given in $[9]$.

The Hausdorff-Besicovitch dimension of the spectrum equals 1 for the cases 1) and 3) above. As is well known (see [13, 24]) the problem of obtaining lower bounds for the Hausdorff-Besicovitch dimension of a set is more challenging than the corresponding problem of obtaining upper bounds. A lower bound for the Hausdorff-Besicovitch dimension of the spectrum for case 2) is a corollary of a formula for the Hausdorff dimension $\operatorname{dim}_{H} \mu_{\xi}$ of a probability measure $\mu_{\xi}$ (see below). The Hausdorff dimension is defined as the number

$$
\operatorname{dim}_{H} \mu_{\xi}=\inf _{E \in B(\mu)} \operatorname{dim}_{H}(E),
$$

where $B(\mu)$ is the family of all Borel supports (not necessarily closed supports) of the probability measure $\mu_{\xi}$. The rest of this section is devoted to the proof of the formula mentioned above. 
Let $\nu$ be a continuous probability measure defined for Borel subsets of the interval $[0,1]$ and let $\Phi$ be the family of cylinders of the $Q_{\infty}$-expansion. The number

$$
H^{\alpha}(M, \Phi, \nu):=\lim _{\varepsilon \rightarrow 0}\left\{\inf _{\nu\left(E_{j}\right) \leq \varepsilon} \sum_{j} \nu^{\alpha}\left(E_{j}\right)\right\},
$$

where $E_{j} \in \Phi$ and $\bigcup_{j} E_{j} \supset M$, is called the Hausdorff-Billingsley $\alpha$-measure of a subset $M \subset[0,1)$ with respect to the family of coverings $\Phi$ and measure $\nu$.

Definition 3.1. The number

$$
\operatorname{dim}_{H}(M, \Phi, \nu)=\inf \left\{\alpha: H^{\alpha}(M, \Phi, \nu)=0\right\}
$$

is called the Hausdorff-Billingsley dimension of the set $M$ with respect to the family of coverings $\Phi$ and measure $\nu$.

If $\nu$ is the Lebesgue measure in the interval $[0,1]$, then $\operatorname{dim}_{H}(M, \Phi, \nu)=\operatorname{dim}_{H}(M, \Phi)$.

Let $\nu$ and $\mu$ be two continuous probability measures defined for Borel sets of the interval $[0,1]$ and let

$$
\Delta_{n}(x)=\Delta_{\alpha_{1}(x) \alpha_{2}(x) \ldots \alpha_{n}(x)}
$$

be a cylinder of rank $n$ of the $Q_{\infty}$-expansion of a number $x$.

The following two results are straightforward corollaries of two classical Billingsley theorems (see [8]) applied to $Q_{\infty}$-expansions.

Lemma 3.1. Let

$$
B=\left\{x: \varliminf_{n \rightarrow \infty} \frac{\ln \nu\left(\Delta_{n}(x)\right)}{\ln \mu\left(\Delta_{n}(x)\right)} \leq \delta\right\} .
$$

Denote by $\Phi$ the family of cylinders of a $Q_{\infty}$-expansion. Then

$$
\operatorname{dim}_{H}(B, \Phi, \mu) \leq \delta
$$

for an arbitrary $\delta \geq 0$.

Lemma 3.2. If

$$
M \subset\left\{x: \varliminf_{n \rightarrow \infty} \frac{\ln \nu\left(\Delta_{n}(x)\right)}{\ln \mu\left(\Delta_{n}(x)\right)} \geq \delta\right\},
$$

then

$$
\operatorname{dim}_{H}(M, \Phi, \mu) \geq \delta \cdot \operatorname{dim}_{H}(M, \Phi, \nu) .
$$

Remark 3.2. To prove Lemmas 3.1 and 3.2, we consider the following probability spaces and stochastic processes that correspond to those considered in [8]. Let

$$
\sigma=\{0,1,2, \ldots\}=: N_{0}
$$

let $\Omega$ be the interval $[0,1)$ and $\mathcal{B}$ be the Borel $\sigma$-algebra of subsets of the interval $[0,1)$; let $\nu$ be an arbitrary continuous probability measure in $\mathcal{B}$. Then, for an arbitrary $\omega \in \Omega$, there exists a unique $Q_{\infty}$-expansion of a number $\omega$ given by

$$
\omega=\Delta_{a_{1}(\omega) a_{2}(\omega) \ldots a_{n}(\omega) \ldots}^{Q_{\infty}} .
$$

Now we introduce the stochastic process

$$
x_{n}(\omega)=a_{n}(\omega), \quad \omega \in \Omega,
$$

where $a_{n}(\omega)$ is the $n$-th digit of the $Q_{\infty}$-expansion of the number $\omega$. For this choice, Billingsley's "abstract cylinders" coincide with $Q_{\infty}$-cylinders.

A similar approach applies to other probability spaces and stochastic processes generated by other decompositions of real numbers, namely by $\widetilde{Q}$-expansions [3], continued fractions, etc. 
Theorem 3.1. Let $\Phi$ be a family of cylinders of a $Q_{\infty}$-expansion that is faithful for the evaluation of the Hausdorff-Besicovitch dimension. If

$$
\sum_{j=1}^{\infty} \frac{\sum_{i=0}^{\infty} p_{i j} \ln ^{2} p_{i j}}{j^{2}}<\infty, \quad \sum_{j=1}^{\infty} \frac{\sum_{i=0}^{\infty} p_{i j} \ln ^{2} q_{i}}{j^{2}}<\infty,
$$

then the Hausdorff dimension of the measure $\mu_{\xi}$ with independent $Q_{\infty}$-digits is equal to

$$
\operatorname{dim}_{H} \mu_{\xi}=\varliminf_{n \rightarrow \infty} \frac{H_{n}}{B_{n}}=: D,
$$

where

$$
\begin{gathered}
H_{n}=\sum_{j=1}^{n} h_{j}, \quad B_{n}=\sum_{j=1}^{n} b_{j}, \\
h_{j}=-\sum_{i=0}^{\infty} p_{i j} \ln p_{i j}, \quad b_{j}=-\sum_{i=0}^{\infty} p_{i j} \ln q_{i} .
\end{gathered}
$$

Proof. Let $x=\Delta_{\alpha_{1}(x) \alpha_{2}(x) \ldots \alpha_{n}(x) \ldots}$ be a random variable such that

$$
\mathrm{P}\left(\alpha_{j}(x)=i\right)=p_{i j}
$$

(that is, the distribution of the random variable $x$ corresponds to the measure $\mu$ ). Consider two auxiliary sequences of random variables $\left\{\eta_{j}(x)\right\}=\left\{\ln p_{\alpha_{j}(x) j}\right\}$ and $\left\{\psi_{j}(x)\right\}=$ $\left\{\ln q_{\alpha_{j}(x)}\right\}$ with the following distributions

\begin{tabular}{|c|c|c|l|c|l|}
\hline$\eta_{j}$ & $\ln p_{0 j}$ & $\ln p_{1 j}$ & $\ldots$ & $\ln p_{k j}$ & $\ldots$ \\
\hline & $p_{0 j}$ & $p_{1 j}$ & $\ldots$ & $p_{k j}$ & $\ldots$ \\
\hline
\end{tabular}

\begin{tabular}{|c|c|c|l|c|l|}
\hline$\psi_{j}$ & $\ln q_{0}$ & $\ln q_{1}$ & $\ldots$ & $\ln q_{k}$ & $\ldots$ \\
\hline & $p_{0 j}$ & $p_{1 j}$ & $\ldots$ & $p_{k j}$ & $\ldots$ \\
\hline
\end{tabular}

Condition (13) implies that the second moments of the random variables $\eta_{j}$ are finite and that the series

$$
\sum_{j=1}^{\infty} \frac{\operatorname{Var} \eta_{j}}{j^{2}}
$$

converges. Then the Kolmogorov strong law of large numbers implies that

$$
\lim _{n \rightarrow \infty} \frac{\left(\eta_{1}+\eta_{2}+\cdots+\eta_{n}\right)-M\left(\eta_{1}+\eta_{2}+\cdots+\eta_{n}\right)}{n}=0
$$

for almost all $x \in[0,1)$ with respect to $\mu_{\xi}$.

Note that

$$
\begin{aligned}
\mathrm{E}\left(\eta_{1}+\eta_{2}+\cdots+\eta_{n}\right) & =\mathrm{E} \eta_{1}+\mathrm{E} \eta_{2}+\cdots+\mathrm{E} \eta_{n} \\
& =-h_{1}-h_{2}+\cdots+\left(-h_{n}\right)=-H_{n}
\end{aligned}
$$

Similarly, condition (13) implies that

$$
\lim _{n \rightarrow \infty} \frac{\left(\psi_{1}+\psi_{2}+\cdots+\psi_{n}\right)-\mathrm{E}\left(\psi_{1}+\psi_{2}+\cdots+\psi_{n}\right)}{n}=0
$$

for almost all $x \in[0,1)$ with respect to $\mu_{\xi}$ and

$$
\begin{aligned}
\mathrm{E}\left(\psi_{1}+\psi_{2}+\cdots+\psi_{n}\right) & =\mathrm{E} \psi_{1}+\mathrm{E} \psi_{2}+\cdots+\mathrm{E} \psi_{n} \\
& =-b_{1}+\left(-b_{2}\right)+\cdots+\left(-b_{n}\right)=-B_{n} .
\end{aligned}
$$


Consider the set

$$
\begin{aligned}
A & =\left\{x: \lim _{n \rightarrow \infty}\left(\frac{\eta_{1}(x)+\eta_{2}(x)+\cdots+\eta_{n}(x)}{\psi_{1}(x)+\psi_{2}(x)+\cdots+\psi_{n}(x)}-\frac{H_{n}}{B_{n}}\right)=0\right\} \\
& =\left\{x: \lim _{n \rightarrow \infty} \frac{\left(\eta_{1}(x)+\eta_{2}(x)+\cdots+\eta_{n}(x)\right)-\frac{H_{n}}{B_{n}}\left(\psi_{1}(x)+\psi_{2}(x)+\cdots+\psi_{n}(x)\right)}{\psi_{1}(x)+\psi_{2}(x)+\cdots+\psi_{n}(x)}=0\right\} \\
& =\left\{x: \lim _{n \rightarrow \infty} \frac{\left(\frac{\eta_{1}(x)+\eta_{2}(x)+\cdots+\eta_{n}(x)+H_{n}}{n}\right)-\frac{H_{n}}{B_{n}}\left(\frac{\psi_{1}(x)+\psi_{2}(x)+\cdots+\psi_{n}(x)+B_{n}}{n}\right)}{\left(\frac{\psi_{1}(x)+\psi_{2}(x)+\cdots+\psi_{n}(x)+B_{n}}{n}\right)-\frac{B_{n}}{n}}=0\right\} .
\end{aligned}
$$

Given a stochastic vector $Q_{\infty}, \max _{j}\left\{q_{j}\right\}$ exists and $\max _{j}\left\{q_{j}\right\}=: \kappa<1$. Thus,

$$
\left|\frac{B_{n}}{n}\right|=\left|\frac{\sum_{j=1}^{n} \sum_{i=0}^{\infty} p_{i j} \ln q_{i}}{n}\right| \geq \frac{|\ln \kappa| n}{n}=|\ln \kappa|>0 .
$$

The classical Gibbs inequality implies that $h_{j} \leq b_{j}$. (One of the well-known proofs of the Gibbs inequality can be found in [16]; recall that the Gibbs inequality is equivalent to the statement that the Kullback-Leibler distance is nonnegative [15].) Hence

$$
\frac{H_{n}}{B_{n}}=\frac{\sum_{j=1}^{n} h_{j}}{\sum_{j=1}^{n} b_{j}} \leq 1 .
$$

Combining equalities (15) and (16) together with inequalities (17) and (18) we see that

$$
\lim _{n \rightarrow \infty} \frac{\left(\frac{\eta_{1}(x)+\eta_{2}(x)+\cdots+\eta_{n}(x)+H_{n}}{n}\right)-\frac{H_{n}}{B_{n}}\left(\frac{\psi_{1}(x)+\psi_{2}(x)+\cdots+\psi_{n}(x)+B_{n}}{n}\right)}{\left(\frac{\psi_{1}(x)+\psi_{2}(x)+\cdots+\psi_{n}(x)+B_{n}}{n}\right)-\frac{B_{n}}{n}}=0
$$

for almost all numbers $x$ of the interval $[0,1)$ with respect to the measure $\mu_{\xi}$. Therefore, $\mu_{\xi}(A)=1$.

Now we consider the sets

$$
\begin{aligned}
A_{1} & =\left\{x: \varliminf_{n \rightarrow \infty}\left(\frac{\eta_{1}(x)+\eta_{2}(x)+\cdots+\eta_{n}(x)}{\psi_{1}(x)+\psi_{2}(x)+\cdots+\psi_{n}(x)}-\frac{H_{n}}{B_{n}}\right)=0\right\}, \\
A_{2} & =\left\{x: \varliminf_{n \rightarrow \infty}\left(\frac{\eta_{1}(x)+\eta_{2}(x)+\cdots+\eta_{n}(x)}{\psi_{1}(x)+\psi_{2}(x)+\cdots+\psi_{n}(x)}\right) \leq \varliminf_{n \rightarrow \infty} \frac{H_{n}}{B_{n}}\right\}, \\
A_{3} & =\left\{x: \varliminf_{n \rightarrow \infty}\left(\frac{\eta_{1}(x)+\eta_{2}(x)+\cdots+\eta_{n}(x)}{\psi_{1}(x)+\psi_{2}(x)+\cdots+\psi_{n}(x)}\right) \geq \varliminf_{n \rightarrow \infty} \frac{H_{n}}{B_{n}}\right\} .
\end{aligned}
$$

It is obvious that $A \subset A_{1}$. Our aim is to show that $A_{1} \subset A_{3}$ and $A \subset A_{2}$. Note that

$$
\varliminf_{n \rightarrow \infty}\left(x_{n}-y_{n}\right) \leq \varliminf_{n \rightarrow \infty}\left(x_{n}\right)-\varliminf_{n \rightarrow \infty}\left(y_{n}\right)
$$

for all sequences of real numbers $\left\{x_{n}\right\}$ and $\left\{y_{n}\right\}$.

If $x \in A_{1}$, then

$$
\begin{aligned}
& \varliminf_{n \rightarrow \infty}\left(\frac{\eta_{1}(x)+\eta_{2}(x)+\cdots+\eta_{n}(x)}{\psi_{1}(x)+\psi_{2}(x)+\cdots+\psi_{n}(x)}\right)-\underline{\lim _{n \rightarrow \infty}} \frac{H_{n}}{B_{n}} \\
& \quad \geq \varliminf_{n \rightarrow \infty}\left(\frac{\eta_{1}(x)+\eta_{2}(x)+\cdots+\eta_{n}(x)}{\psi_{1}(x)+\psi_{2}(x)+\cdots+\psi_{n}(x)}-\frac{H_{n}}{B_{n}}\right)=0 .
\end{aligned}
$$

Thus, $x \in A_{3}$.

If $x \in A$, then

$$
\lim _{n \rightarrow \infty}\left(\frac{\eta_{1}(x)+\eta_{2}(x)+\cdots+\eta_{n}(x)}{\psi_{1}(x)+\psi_{2}(x)+\cdots+\psi_{n}(x)}-\frac{H_{n}}{B_{n}}\right)=0
$$


and

$$
\begin{aligned}
\varliminf_{n \rightarrow \infty} & \frac{H_{n}}{B_{n}}-\varliminf_{n \rightarrow \infty}\left(\frac{\eta_{1}(x)+\eta_{2}(x)+\cdots+\eta_{n}(x)}{\psi_{1}(x)+\psi_{2}(x)+\cdots+\psi_{n}(x)}\right) \\
& \geq \varliminf_{n \rightarrow \infty}\left(\frac{H_{n}}{B_{n}}-\frac{\eta_{1}(x)+\eta_{2}(x)+\cdots+\eta_{n}(x)}{\psi_{1}(x)+\psi_{2}(x)+\cdots+\psi_{n}(x)}\right) \\
& =\varliminf_{n \rightarrow \infty}\left(\frac{\eta_{1}(x)+\eta_{2}(x)+\cdots+\eta_{n}(x)}{\psi_{1}(x)+\psi_{2}(x)+\cdots+\psi_{n}(x)}-\frac{H_{n}}{B_{n}}\right)=0 .
\end{aligned}
$$

Thus, $x \in A_{2}$.

Since

$$
A \subset A_{2}=\left\{x: \varliminf_{n \rightarrow \infty} \frac{\ln \mu_{\xi}\left(\Delta_{n}(x)\right)}{\ln \lambda\left(\Delta_{n}(x)\right)} \leq D\right\},
$$

Lemma 3.1 implies that

$$
\operatorname{dim}_{H}(A, \Phi)=\operatorname{dim}_{H}(A, \Phi, \lambda) \leq D .
$$

Further, since

$$
A \subset A_{3}=\left\{x: \varliminf_{n \rightarrow \infty} \frac{\ln \mu_{\xi}\left(\Delta_{n}(x)\right)}{\ln \lambda\left(\Delta_{n}(x)\right)} \geq D\right\},
$$

Lemma 3.2 implies that

$$
\operatorname{dim}_{H}(A, \Phi) \geq D \cdot \operatorname{dim}_{H}\left(A, \Phi, \mu_{\xi}\right)=D .
$$

Thus, $\operatorname{dim}_{H}(A, \Phi)=D$.

It remains to prove that the set $A$ is a minimal support of the measure $\mu_{\xi}$ (the minimality of a support is understood in the sense of the minimum of its dimension). Let $C$ be an arbitrary support of the measure $\mu_{\xi}$. Then the set $C_{1}=C \cap A$ is also a support of $\mu_{\xi}$ and $C_{1} \subset C$. Hence $\operatorname{dim}_{H}\left(C_{1}, \Phi\right) \leq \operatorname{dim}_{H}(C, \Phi)$. Next we show that $\operatorname{dim}_{H}\left(C_{1}, \Phi\right)=\operatorname{dim}_{H}(A, \Phi)$.

Since $C_{1} \subset A$, we get $\operatorname{dim}_{H}\left(C_{1}, \Phi\right) \leq \operatorname{dim}_{H}(A, \Phi)$. On the other hand,

$$
C_{1} \subset A \subset A_{3}=\left\{x: \varliminf_{n \rightarrow \infty} \frac{\ln \mu_{\xi}\left(\Delta_{n}(x)\right)}{\ln \lambda\left(\Delta_{n}(x)\right)} \geq D\right\}
$$

and Lemma 3.2 implies that $\operatorname{dim}_{H}\left(C_{1}, \Phi\right) \geq D$.

Therefore, $\operatorname{dim}_{H}\left(C_{1}, \Phi\right)=D=\operatorname{dim}_{H}(A, \Phi)$. Since $\Phi$ is a faithful family of cylinders of the $Q_{\infty}$-expansion, $\operatorname{dim}_{H}(A, \Phi)=\operatorname{dim}_{H}(A)$, whence we conclude that $A$ is a minimal support of the probability measure $\mu_{\xi}$ (the minimality is understood in the sense of minimality of the Hausdorff-Besicovitch dimension).

As a corollary of Theorem 3.1 we obtain a lower bound for the Hausdorff-Besicovitch dimension of the spectrum of a probability measure with independent $Q_{\infty}$-digits discussed above. Let $N_{j}=\left\{i: p_{i j} \neq 0\right\}$ and

$$
q_{j}^{*}=\sum_{i \in N_{j}} q_{i}, \quad j \in \mathbf{N} .
$$

Theorem 3.2. Let $\Phi$ be a faithful family of cylinders of the $Q_{\infty}$-expansion for the evaluation of the Hausdorff-Besicovitch dimension. Assume that

$$
\sum_{j=1}^{\infty} \frac{\sum_{i \in N_{j}} \frac{q_{i}}{q_{j}^{*}} \ln ^{2} q_{i}}{j^{2}}<\infty
$$

Then

$$
\operatorname{dim}_{H}\left(S_{\xi}\right) \geq \lim _{n \rightarrow \infty} \frac{H_{n}^{*}}{B_{n}^{*}}
$$


where

$$
\begin{array}{ll}
H_{n}^{*}=\sum_{j=1}^{n} h_{j}^{*}, \quad h_{j}^{*}=-\sum_{i \in N_{j}} \frac{q_{i}}{q_{j}^{*}} \cdot \ln \frac{q_{i}}{q_{j}^{*}}, \\
B_{n}^{*}=\sum_{j=1}^{n} b_{j}^{*}, & b_{j}^{*}=-\frac{1}{q_{j}^{*}} \sum_{i \in N_{j}} q_{i} \cdot \ln q_{i} .
\end{array}
$$

Proof. The distribution of the random variable $\xi$ is determined by the matrix $P=\left\|p_{i k}\right\|$ and stochastic vector $Q_{\infty}$. Starting with the stochastic vector $Q_{\infty}$ we construct an auxiliary random variable $\xi^{a}$ with independent $Q_{\infty}$-digits, namely we put

$$
p_{i j}^{a}= \begin{cases}0, & \text { if } p_{i j}=0, \\ q_{i} / q_{j}^{*}, & \text { if } p_{i j}>0 .\end{cases}
$$

Let $S_{\xi^{a}}$ be the spectrum of the distribution of the random variable $\xi^{a}$. The spectra $S_{\xi^{a}}$ and $S_{\xi}$ coincide, since $q_{i}^{a}=q_{i}$ and $p_{i j}^{a}=0$ if and only if $p_{i j}=0$. Since the spectrum of the measure is only one of the possible Borel supports defined as a minimal closed support, then

$$
\operatorname{dim}_{H} \mu_{\xi^{a}} \leq \operatorname{dim}_{H}\left(S_{\xi^{a}}\right) .
$$

Condition (19) implies that all the assumptions of the preceding theorem hold for the random variable $\xi^{a}$. Applying Theorem 3.1 and evaluating the Hausdorff dimension of the distribution of the random variable $\xi^{a}$, we get

$$
\operatorname{dim}_{H}\left(S_{\xi}\right) \geq \operatorname{dim}_{H} \mu_{\xi^{a}}=\underline{\lim }_{n \rightarrow \infty} H_{n}^{*} / B_{n}^{*} .
$$

\section{ACKNowledgement}

The authors are grateful to reviewers for a careful reading of the manuscript and their suggestions lead to an improvement of the paper.

\section{BIBLIOGRAPHY}

1. S. Albeverio and G. Torbin, Fractal properties of singularly continuous probability distributions with independent $Q^{*}$-digits, Bull. Sci. Math. 129 (2005), no. 4, 356-367. MR2134126 (2006b:28013)

2. S. Albeverio, Yu. Kondratiev, R. Nikiforov, and G. Torbin, On fractal phenomena connected with infinite linear IFS and related singular probability measures, J. London Math. Soc. (submitted)

3. S. Albeverio, V. Koshmanenko, M. Pratsiovytyi, and G. Torbin, On fine structure of singularly continuous probability measures and random variables with independent $\widetilde{Q}$-symbols, Meth. Funct. Anal. Topology 17 (2011), no. 2, 97-111. MR2849470 (2012g:60006)

4. S. Albeverio, V. Koval, M. Pratsiovytyi, and G. Torbin, On classification of singular measures and fractal properties of quasi-self-affine measures in $R^{2}$, Random Oper. Stoch. Equations 16 (2008), no. 2, 181-211. MR2446437 (2010b:28008)

5. A. Besicovitch, On existence of subsets of finite measure of sets of infinite measure, Indag. Math. 14 (1952), 339-344. MR0048540(14:28e)

6. M. Bernardi and C. Bondioli, On some dimension problems for self-affine fractals, J. Anal. Appl. 18 (1999), no. 3, 733-751. MR1718162 (2000j:28004)

7. P. Billingsley, Ergodic Theory and Information, John Wiley and Sons, New York, 1965. MR0192027 (33:254)

8. P. Billingsley, Hausdorff dimension in probability theory II, Ill. J. Math. 5 (1961), 291-298. MR0120339(22:11094)

9. B. H. Bissinger, A generalization of continued fractions, Bull. Amer. Math. Soc. 50 (1944), 868-876. MR0011338 (6:150h)

10. S. D. Chatterji, Certain induced measures and the fractional dimensions of their "supports", Z. Wahrscheinlichkeitstheorie verw. Geb. 3 (1964), 184-192. MR0174691 (30:4891) 
11. C. D. Cutler, A note on equivalent interval covering systems for Hausdorff dimension on $R$, Internat. J. Math. Math. Sci. 4 (1988), 643-650. MR959443 (89h:28008)

12. C. I. Everett, Representations for real numbers, Bull. Amer. Math. Soc. 52 (1946), 861-869. $\operatorname{MR} 0018221(8: 259 \mathrm{c})$

13. K. J. Falconer, Fractal Geometry: Mathematical Foundations and Applications, Wiley, Chichester, 1990. MR1102677 (92j:28008)

14. J. E. Hutchinson, Fractals and self similarity, Indiana Univ. Math. J. 30 (1981), 713-747. MR625600 (82h:49026)

15. S. Kullback and R. A Leibler, On information and sufficiency, Ann. Math. Statist. 22 (1951), 79-86. MR0039968(12:623a)

16. R. O. Nikiforov and G. M. Torbin, Ergodic properties of $Q_{\infty}$-expansions and fractal properties of probability measures with independent $Q_{\infty}$-symbols, Proceedings of the National Dragomanov Pedagogical University, Physics and Mathematics 9 (2008), 80-103. (Ukrainian)

17. Yu. Peres and G. Torbin, Continued fractions and dimensional gaps. (in preparation)

18. M. V. Pratsevytyı̆, Fractal Approach in Studies of Singular Distributions, Dragomanov National Pedagogical University Press, Kyiv, 1998. (Ukrainian)

19. M. V. Pratsevytyı̆ and O. L. Leshchins'kyı̆, Properties of random variables defined in terms of the distributions of elements of their $\tilde{Q}_{\infty}$-representation, Teor. Imovir. Matem. Statyst. 57 (1997), 134-140; English transl. in Theory Probab. Math. Statist. 57 (1998), 143-148.

20. M. V. Pratsevytyı̆ and G. M. Torbin, On a classification of one dimensional singularly continuous probability measures with respect to their spectral properties, Proceedings of the National Dragomanov Pedagogical University, Physics and Mathematics 7 (2006), 140-151. (Ukrainian)

21. M. V. Pratsevyty̌, G. M. Torbin, An analytic (symbol) representation of continuous transformations of $R^{1}$ that preserve the Hausdorff-Besicovitch dimension, Proceedings of the National Dragomanov Pedagogical University, Physics and Mathematics 4 (2003), 207-215. (Ukrainian)

22. A. Renyi, Representations for real numbers and their ergodic properties, Acta Math. Sci. Hungar. 8 (1957), 477-493. MR0097374 (20:3843)

23. G. M. Torbin, Multifractal analysis of singularly continuous probability measures, Ukr. Matem. Zh. 57 (2005), no. 5, 706-721; English transl. in Ukr. Math. J. 57 (2005), no. 5, 837-857. MR2209816(2007f:28010)

24. A. F. Turbin and M. V. Pratsevytyı̆, Fractal sets, functions, distributions, "Naukova Dumka", Kiev, 1992. (Russian) MR.1353239 (96f:28010)

Department of Higher Mathematics, Dragomanov National Pedagogical University, PiroGova Street 9, Kyiv 01130, Ukraine

E-mail address: rnikiforov@gmail.com

Department of Mathematical Analysis and Differential Equations, Dragomanov National Pedagogical University, Pirogova Street 9, Kyiv 01130, Ukraine - and - Department of FraCtal Analysis, Institute for Mathematics, National Academy of Science of Ukraine, TereshchenKIVs'Ka Street 3, Kyiv 01130, Ukraine

E-mail address: torbin7@gmail.com

E-mail address: torbin@imath.kiev.ua 